\title{
Diagnosis and grading of radiographic osteoporotic vertebral deformity by general radiologists after a brief self-learning period
}

\author{
Yì Xiáng J. Wáng ${ }^{1}$, Wei-Hong Liu ${ }^{2}$, Davide Diacinti ${ }^{3,4}$, Da-Wei Yang ${ }^{5}$, Antonio Iannacone ${ }^{6}$, \\ Xiao-Rong Wang ${ }^{7}$, Endi Kripa ${ }^{6}$, Nazmi Che-Nordin ${ }^{1}$, Daniele Diacinti ${ }^{6}$
}

${ }^{1}$ Department of Imaging and Interventional Radiology, Faculty of Medicine, The Chinese University of Hong Kong, Shatin, New Territories, Hong Kong, China; ${ }^{2}$ Department of Radiology, General Hospital of China Resources \& Wuhan Iron and Steel Corporation, Wuhan, China; ${ }^{3}$ Department of Oral and MaxilloFacial Sciences, Umberto I Hospital, Sapienza University of Rome, Rome, Italy; ${ }^{4}$ Department of Diagnostic and Molecular Imaging, Radiology and Radiotherapy, University Foundation Hospital Tor Vergata, Rome, Italy; ${ }^{5}$ Department of Radiology, Beijing Friendship Hospital, Capital Medical University, Beijing, China; ${ }^{6}$ Department of Radiological Sciences, Oncology and Pathology, Sapienza University of Rome, Rome, Italy; ${ }^{7}$ Department of Radiology, Ningbo First Hospital, Ningbo, China

Contributions: (I) Conception and design: YXJ Wáng; (II) Administrative support: N Che-Nordin, Daniele Diacinti; (III) Provision of study materials or patients: YXJ Wáng, Daniele Diacinti; (IV) Collection and assembly of data: YXJ Wáng, N Che-Nordin, Daniele Diacinti; (V) Data analysis and interpretation: All authors; (VI) Manuscript writing: All authors; (VII) Final approval of manuscript: All authors.

Correspondence to: Dr. Yì Xiáng J. Wáng. Department of Imaging and Interventional Radiology, the Chinese University of Hong Kong, Shatin, New Territories, Hong Kong, China. Email: yixiang_wang@cuhk.edu.hk.

Background: The expanded semi-quantitative (eSQ) osteoporotic vertebral deformity (OVD) classification has minimal, mild, moderate, moderately-severe, severe, and collapsed grades with $<20 \%, 20-25 \%$, $>25 \%-$ $1 / 3,>1 / 3-40 \%,>40 \%-2 / 3,>2 / 3$ vertebral height loss respectively. This study evaluates the performance of using this grading criterion by radiology readers who did not have former training in OVD assessment.

Methods: Spine radiographs of 44 elderly women with 278 normal appearing vertebrae and 65 OVDs were selected, with two senior readers agreed the reference reading. Three readers from Italy and three readers from China were invited to evaluate these radiographs after reading five reference articles including one detailing eSQ criteria with illustrative examples. Before the second round of reading, the readers were asked to read an additional explanatory document. For the readers in Italy an additional on-line demonstration was given on how to measure vertebral height loss in another five cases of OVD. Two Chinese readers had a third round of reading after a 90 minutes' on-line lecture.

Results: The final absolute agreement rate with the reference reading (i.e., exactly the same grading as the reference) ranged between $46.2 \%$ to $68.2 \%$ for the six readers, and the final relative agreement (with one eSQ grade difference allowed) ranged between $78.5 \%$ to $92.5 \%$. The $>1$ grade disagreement rate was all below $11 \%$, and mostly below $7 \%$. The missed OVD were mostly minimal grade. The rate for missing a $\geq$ mild OVD was $<4.5 \%$, and false positive rate was generally $<1.4 \%$ among the final reading. If the minimal grade was removed and the remaining gradings were converted to Genant's semi-quantitative (GSQ) grading, the mean kappa values against the reference reading for SQ grades-1,2,3 were 0.813, 0.814, and 0.916 respectively.

Conclusions: This study demonstrates good performance of the six learner readers for assessing radiographic after a brief self-learning period.

Keywords: Osteoporosis; osteoporotic vertebral deformity (OVD); osteoporotic vertebral fracture (OVF); spine; grading

Submitted Jul 07, 2020. Accepted for publication Aug 06, 2020.

doi: $10.21037 /$ jtd-20-2379

View this article at: http://dx.doi.org/10.21037/jtd-20-2379 


\section{Introduction}

Osteoporotic vertebral fracture (OVF) is the most common osteoporotic fracture. A vertebral fracture, after minor trauma, is a hallmark of osteoporosis. OVF predispose to both further fracturing and fracture cascade (1-5). OVF can be associated with decreases in trunk extension torque, spinal motion, functional reach, mobility skills and walking distance. OVF may also influence mortality because of its association with chronic back pain, immobility and change in posture, which may increase the risk of infection and death (2). Appropriate interventions for OVF can reduce the occurrence of hip fractures, preventing further OVF and reducing pain and related disabilities. It is important to identify and report OVF, so that appropriate investigation and treatment can be instigated. However, OVF may often be relatively asymptomatic so that, in the absence of localizing symptoms, spinal fracture status is usually determined by obtaining lateral radiographs of the spine either by radiograph or by dual-energy $\mathrm{X}$-ray absorptiometry (DXA) devices operating in imaging mode (4).

The Genant's semi-quantitative (GSQ) criteria is currently the most used approach for identifying OVF (5-7). The qualitative diagnosis with radiological knowledge helps to minimize false positive readings due to vertebral physiological wedging, degenerative wedging, and other congenital anomalies. Radiological evaluation can also detect subtle changes that may be clinical important, such as endplate and/or cortex fracture (ECF) (8-13). According to Genant et al., a vertebra body (VB) is graded on visual inspection of the anterior, middle, or posterior heights as normal (grade-0), mildly deformed (grade-1, a 20-25\% reduction in one of the three heights and a reduction in area of $10-20 \%$ ), moderately deformed (grade-2, a $25-40 \%$ reduction in any height and a reduction in area of 20-40\%) and severely deformed (grade- 3 , a $40 \%$ or more reduction in height and area). In addition to the height reduction estimation, emphasis is also placed on the radiological evaluation of osteoporotic vertebral deformity (OVD). However, unless there is a face-to-face training with experienced readers, it has been well documented that it is difficult to apply GSQ criteria by only reading the text description of Genant et al. (5,14-17). For example, despite that a standardized protocol of radiograph acquisition techniques and of interpretation criteria was applied, Diacinti et al. (16) reported a study that, among 562 OVFs identified by radiologist readers in peripheral hospitals, 102 were classified as normal vertebrae by the experienced radiologist readers in a central hospital; while 205 OVFs were incorrectly evaluated by local readings as (false) negatives.

Built upon the GSQ criteria, Wáng et al. (18) recently proposed an expanded semi-quantitative (eSQ) OVD classification with the following features: (I) minimal grade is OVDs with $<20 \%$ VB height loss; (II) eSQ mild grade is the same as GSQ mild grade $(20-25 \%$ height loss); (III) GSQ moderate grade is subdivided into eSQ moderate grade ( $\mathrm{mod} / \mathrm{m},>25 \%-1 / 3$ height loss) and eSQ moderately-severe grade ( $\mathrm{mod} / \mathrm{s},>1 / 3-40 \%$ height loss); (IV) GSQ severe grade is subdivided into eSQ severe grade ( $>40 \%-2 / 3$ height loss) and eSQ collapsed grade (with $>2 / 3$ height loss). The eSQ criteria introduce a formal minimal grade, equivalent to GSQ grade-0.5, referring to radiological OVDs with less than $20 \%$ height loss. While GSQ grade-0.5 OVDs are common, it is less reported and discussed in literature (6). eSQ minimal grade can be clinically relevant specially when there is associated ECF $(8-12,19)$. GSQ moderate is subdivided into eSQ $\mathrm{mod} / \mathrm{m}$ and $\mathrm{mod} / \mathrm{s}$ grades because OVDs with $>1 / 3$ height loss are always associated with positive ECF sign radiographically (this is the reason for such a subdivision) $(12,20,21)$. It has been noted that compared with VBs without OVD, ECF(-) GSQ grade-1 and grade-2 OVDs do not have a higher short-term future risk for new incident OVD. Within the same GSQ mild/moderate OVD grades, compared with the subjects without ECF, the subjects with ECF are associated with a higher short-term future risk of OVD progression and new incident OVD (12). A subdivision GSQ grade-3 will allow recording of severe OVD progressions (such as a $45 \%$ height loss progresses to $75 \%$ height loss). Wáng et al. (18) defined how VB height loss is measured, and also provide graphic illustration of 36 cases with radiographic OVD.

This study aims to test the hypothesis that, since Wáng et al.'s article defines how VB height loss is measured and users can refer to the 36 cases illustrated in the article, a general radiologist can self-learn by reading a couple of key reference papers including Wáng et al.'s, and performs well in evaluating radiographic OVD without face-to-face coaching from experienced readers.

\section{Methods}

This study was approved by the local ethics committees of authors' institutions. Informed consent for additional analysis of radiographs in this study was waived. The 
Table 1 Vertebral height loss criteria for Genant semi-quantitative (GSQ) grading and extended semi-quantitative grading (eSQ)*

\begin{tabular}{|c|c|c|c|c|c|c|}
\hline Variable & \multicolumn{6}{|c|}{ Extent of vertebral height loss } \\
\hline Genant SQ & Grade-0.5 & Grade-1 & \multicolumn{2}{|c|}{ Grade-2 } & \multicolumn{2}{|c|}{ Grade-3 } \\
\hline Genant SQ & & Mild & \multicolumn{2}{|c|}{ Moderate } & \multicolumn{2}{|c|}{ Severe } \\
\hline Extended SQ & Grade-1 & Grade-2 & Grade-3 & Grade-4 & Grade-5 & Grade-6 \\
\hline
\end{tabular}

${ }^{*}$, vertebrae with normal radiographical morphology is noted as grade- 0 .

thoracic and/or lumbar spine radiograph of 29 Chinese cases and 15 Italian cases were randomly selected from senior authors' study database, with in total $343 \mathrm{VBs}$ visible on the radiographs. All subjects were females and aged $>55$ years old, and according to the two senior authors (YXJW, DD) had at least one OVD per subject according to the eSQ criteria. The two senior authors had been involved in the OVD/OVF evaluation in the past, but they did not have face-to-face interaction. These two senior authors initially evaluated the 44 cases independently according to eSQ criteria (Table 1), and found they agreed in 278 VBs without OVD, and $65 \mathrm{VBs}$ with OVD among them there were 7 OVDs with disagreement by one eSQ grade (none had disagreement by > one grade), and one VB with upper endplate Schmorl's node co-existing with ambiguous minimal grade OVD. A second-round reading resolved disagreement in 4 OVDs, and with remaining three OVDs considered two grades were acceptable for each VB (e.g., an OVD with a measured height loss of $39 \%$ was considered acceptable for both mod/s and severe grades). The agreed reading of these two readers were then regarded as the reference. In total, there were 10 minimal, 11 mild, $21 \mathrm{mod} / \mathrm{m}, 7 \mathrm{mod} / \mathrm{s}, 17$ severe, and 3 collapsed grade OVDs; while one OVD was either minimal grade or mild grades, two OVDs were either mod/s grade or severe grades; and for additional one $\mathrm{VB}$, both minimal grade OVD or without OVD were considered acceptable.

This study recruited three young general radiologist readers from Italy (I1, I2, I3) and three young general radiologists from China $(\mathrm{C} 1, \mathrm{C} 2, \mathrm{C} 3)$. They were asked not to communicate on the study topics during the testing period. None of them were subspecialized in musculoskeletal radiology and none of them participated special clinical program in OVD assessment. These 6 radiologists were asked to read the three papers of references $5,18,22$ as the primary teaching materials $(5,18,22)$, and two papers of references 23,24 as supplementary teaching materials $(23,24)$. Among them Wáng et al.'s article is the key teaching material (18). Szulc's letter gives a good introduction of how to identify an OVD (5), and Wang \& Che-Nordin's letter explains Genant's SQ criteria (22). The two supplementary papers mainly provide teaching material on ECF identification, and in the meantime provide more examples of OVD $(23,24)$. After reading the teaching articles, the 6 readers were asked to evaluate the 44 study cases' blinded radiographs, identifying the VBs with OVD and their eSQ grading. The VB dimension measurement was done using the software MicroDicom DICOM viewer (https://www.microdicom.com/). The measurement was computer screen-captured and feedback to the two senior authors. For second round of reading, the readers in China were asked to read an additional document (supplementary document at: http://fp.amegroups.cn/cms/fae 5c5f22eef2 1ad80f0b8003d120288/JTD-20-2379-1.pdf), and then to evaluate the radiographs again. This additional document was prepared after senior author-YXJW reviewed the reading results of the 6 readers with feedback of computer screen-captures. This document comments on the common errors of the first round of test readings, but no specific case or example was discussed. For the readers in Italy, in addition to reading this document, senior author-DD did an on-line demonstration of how to measure vertebral height loss in another 5 five cases of OVD which the readers in Italy measured two days later, and then experienced senior author-DD corrected their measurements and gave feedback to the three Italian readers. After that, the Italian readers evaluated the radiographs again. Two Chinese readers $(\mathrm{C} 2, \mathrm{C} 3)$ had a third round of reading. Via an online platform, senior author-YXJW spent 90 minutes to explain Wáng et al.'s article (18); and explained his approach to evaluate and grade OVD. The content of this lecture focuses on the important points of what were already on 
Table 2 Final agreement (vertebral number and percentage) between the six learner readers and reference reading

\begin{tabular}{|c|c|c|c|c|c|}
\hline Variable & OVD absolute agree* & OVD relative agree* & OVD disagree by $>1$ grade $^{*}$ & $\geq$ mild OVD missed $^{\star}$ & False (+) OVD \\
\hline 12 & $39(60 \%)$ & $54(83.1 \%)$ & $4(6.2 \%)$ & 0 & 0 \\
\hline 13 & $30(46.2 \%)$ & $51(78.5 \%)$ & $7(10.8 \%)$ & 0 & 0 \\
\hline $\mathrm{C} 1$ & $42(63.6 \%)$ & $60(90.9 \%)$ & $4(6.1 \%)$ & 0 & $4(1.4 \%)$ \\
\hline C3 & $42(63.6 \%)$ & $59(89.4 \%)$ & $4(6.1 \%)$ & $2(3.0 \%)$ & $2(0.7 \%)$ \\
\hline
\end{tabular}

OVD absolute agree: exactly the same grading as the reference reading; OVD relative agree: compared with the reference reading, one grade difference is allowed; OVD disagree by $>1$ grade: disagreement by more than one grade, for example, a mild grade is marked as $\mathrm{mod} / \mathrm{s}$ grade; $\geq$ mild OVD missed: mild grade OVD were missed with C2 missed one severe OVD (due to oversight). *, out of total true OVD; " falsely marked as OVD out of total vertebrae with no OVD. OVD, osteoporotic vertebral deformity.

the teaching materials, with no additional case or example provided.

For statistical analysis, Kappa coefficient was used for to measure inter-reader reliability for categorical items. The performance of the 6 general radiologists was assessed with agreement criteria comparing with the reference reading by the two senior authors.

\section{Results}

The key indicators of final agreement between the six learner readers and reference reading are summarized in Table 2, and the detailed agreements are shown in Table 3. Table 2 shows the final absolute agreement rate (i.e., exactly the same grading as the reference) ranged between $46.2 \%$ to $68.2 \%$, and the final relative agreement (with one grade difference allowed) ranged between $78.5 \%$ to $92.5 \%$. The $>1$ grade disagreement in grading was all below $11 \%$. The missed OVDs were mostly minimal grade. The rate for missing a $\geq$ mild OVD was $<4.5 \%$. One severe OVD was missed by Chinese reader-2; however, it was an oversight rather than misjudgement which was confirmed after the study completed. The false positive rate was generally $<1.4 \%$ among the final reading, and all were marked as minimal grade. If grading was disregarded and a VB was only considered either OVD positive or negative, according to eSQ the positive agreement was $90.8 \%, 89.2 \%, 89.2 \%$, $96.9 \%, 92.4 \%$, and $96.9 \%$ respectively for the six learner readers; and for negative VBs, the agreement was $99.6 \%$, $100 \%, 100 \%, 98.2 \%, 98.9 \%$, and $98.9 \%$ respectively.

If the eSQ minimal grade was removed, eSQ $\mathrm{mod} / \mathrm{m}$ and $\mathrm{mod} / \mathrm{s}$ grades were merged into a single moderate grade, and eSQ severe and collapsed grades were merged into severe grade, the reading results were then converted into GSQ results. The GSQ results are shown in Table 4. The mean kappa value for GSQ grades-1,2,3 was $0.813,0.814$, and 0.916 , respectively.

For the second round of reading, the three Italian readers and Chinese reader-1 performed the same as the 1st round of reading, while Chinese reader- 2 and -3 improved notably. Chinese reader-2 further improved during the $3 \mathrm{rd}$ round of reading.

\section{Discussion}

Despite years' of research, the radiographic criteria for diagnosing osteoporotic $\mathrm{VF}$ and its grading remain debated (14,25-28). Recent evidences favor radiological diagnosis over OVD's morphometric criteria $(14,27,28)$. For example, Leidig-Bruckner et al. (27) applied an algorithm for radiological differential classification (RDC), and their RDC's reading were compared with BMD measurement as well as Eastell and McCloskey's morphometric criteria $(29,30)$. In women, 31-68\% of cases with morphometric OVD were classified by RDC as non-osteoporotic. On the other hand, up to $48 \%$ OVD in women were missed by morphometry. Although OVD by most definitions were significantly and inversely related to $\mathrm{BMD}$, a stronger association existed between BMD and osteoporotic fractures defined by RDC (17). In the early 1990s, Genant et al. (6) proposed the GSQ criteria. Though the GSQ is commonly used, it is associated with two major difficulties. The first is to decide whether a mild OVD exists for a VB, or it is only a degenerative wedging. The second is the precise grading. These difficulties may not be so an issue for clinical practice; but can cause problems for epidemiological 


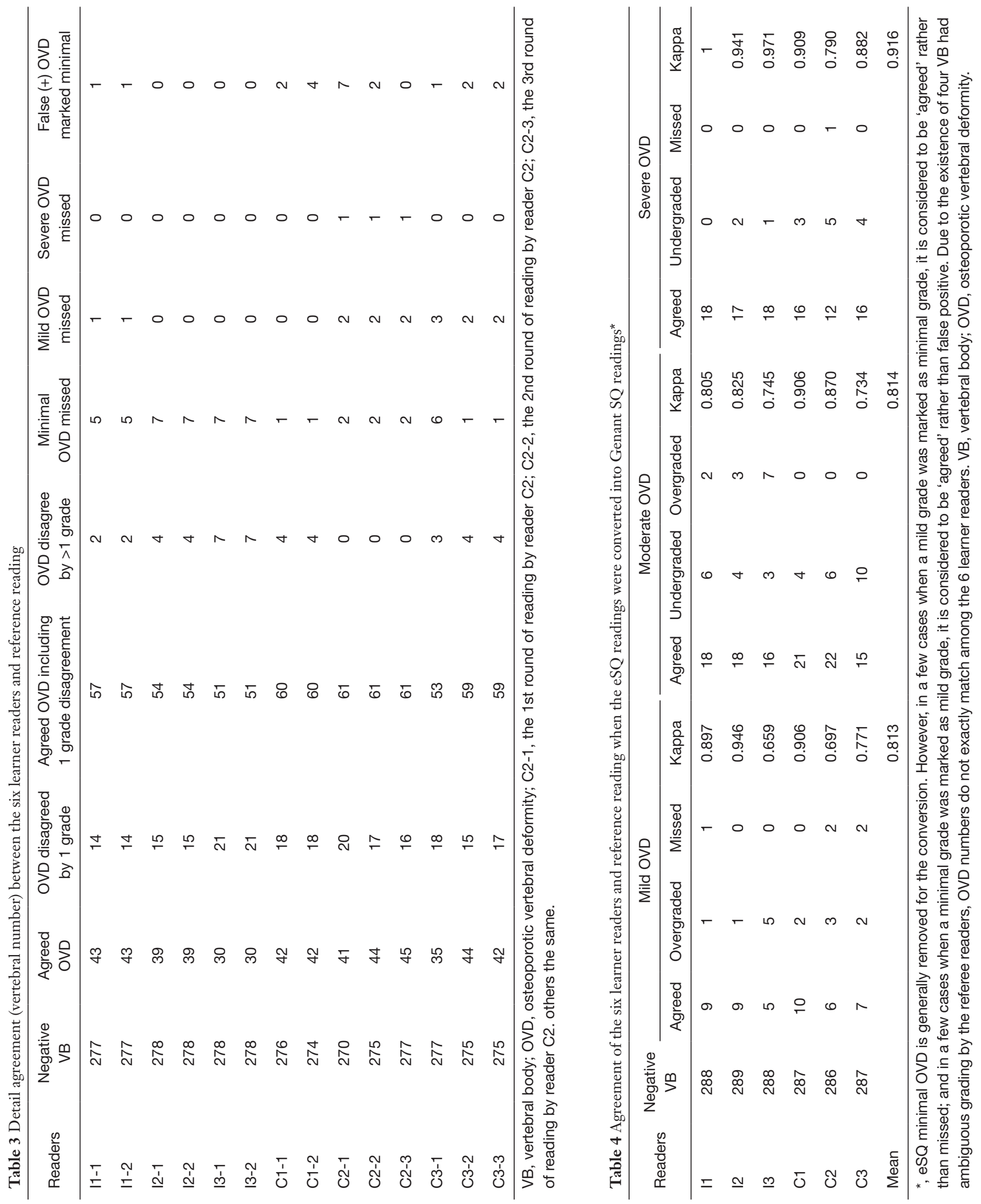


studies, and particularly for cross-center results comparison. GSQ has been generally perceived difficult to implement by those readers who have not received training from experienced readers. The agreement among readers for OVD GSQ grading can be poor (14). GSQ method relies on estimate of vertebral dimensions, which is a potential source of observer error. Though it has not been specifically noted, it is implied that the posterior VB height is used as the reference to estimate middle/anterior height loss. However, to compare the height loss of a vertebra with its adjacent vertebrae is more reasonable (18), as the ratio of anterior height to posterior height varies among different vertebral levels. Additionally, due to the existence of vertebra's posterior lip (uncinate process), the posterior height measure can cause inconsistencies.

Wáng et al.'s work tries to address the difficulties of GSQ. In addition to proposing the eSQ scheme with the perceived advantages, Wáng et al.'s paper also defines the way the VB height loss is measured. It may help to resolve difference by individual readers to facilitate consensus reading. For example, a 38\% height loss can be read as GSQ moderate grade by one reader and GSQ severe grade by another reader, which would be difficult to reconcile without a consistent measurement. Since original GSQ criteria were primarily explained by text $(6,7)$, rather than by examples, Wáng et al.'s paper provides 36 illustrative examples with eSQ and GSQ grading. Though Genant et al. described GSQ grade-0.5 (a qualitative OVD with less than $20 \%$ height loss) (6), it was rarely used and reported, and to our knowledge many GSQ grade-0.5 OVDs might have been assigned as SQ grade-1 in literature by some readers (and may be ignored by other readers) $(6,31)$. The introduction of a formal eSQ grade-1 may solve the problem when a qualitative OVD exists but it does not look like achieving the threshold of $\geq 20 \%$ vertebral height loss. Thus, the reader is not forced to make a 'YES' or 'NO' choice. It has been noted that even some of the GSQ mild grade VD may not be true OVF $(32,33)$. The eSQ criteria will allow flexibility in application, for example, a researcher may decide to exclude all eSQ minimal grade OVDs, or may only account eSQ minimal grade OVDs with positive ECF sign (18).

We consider the results of this study being satisfactory. With some degree of commitment, the initial reading results can be improved as shown by the 1 st and 2 nd round results of readers $\mathrm{C} 2$ and $\mathrm{C} 3$. A precise comparison with previous results would be difficult. Firstly, the readers in this study would have guessed that at least an OVD exists per subject (though they were not informed as such). In real practice, readers have to read two radiographs (one thoracic spine radiograph and one lumbar spine radiograph) per subject, while, with this study, focused radiographs with on average $8 \mathrm{VBs}$ each were provided. However, results in this study compare favorably with previous publications. Recently, Diacinti et al. (16) reported a study to evaluate the diagnostic accuracy of OVF by radiologist readers in peripheral hospitals with ordinary experience and using the GSQ criteria, their readings were compared with the reference reading by two experienced central radiologists. It was noted that $30.8 \%$ of GSQ grade-1 OVFs were mis-diagnosed by the general radiologists, despite that a standardized protocol of radiograph acquisition techniques and interpretation criteria was applied. In that study, among $562 \mathrm{VFs}$ identified by general radiologist readers, 102 were classified as normal vertebrae by the central readers; while 205 vertebrae were incorrectly evaluated by general radiologist as not presenting OVFs. Fechtenbaum et al. (15) reported the agreement for OVF diagnosis among the 390 vertebrae in male patients between thirteen experimented rheumatologists. After the group discussed in a workshop and established an algorithm of VF diagnosis, the number of OVF detected varies from 18 to 59 . The mean concordance percentage (negative or positive agreement) between experts at the vertebral level ranged from $83.1 \%$ to $97.9 \%$. Wu et al. (34) reported three experienced readers' inter-reader kappa scores based on the dichotomous fracture/nonfracture decision, which ranged from 0.84 to 0.87 between the individual readings and the consensus reading, and 0.69 agreement between two readers. Buckens et al. (35) assessed the agreement of four observers with different levels of experience, and sagittal reformatted CT images were used. For fracture presence the interobserver kappa scores ranged from 0.56 to 0.81 . For GSQ grade-3 OVF, the interobserver kappa scores ranged from 0.73 to 0.88 .

One aspect of the results in this study which surprised the senior authors is that the on-line coaching did not substantially improved the reading results. This may suggest that a 1-2 hours on-line interaction is insufficient to systematically improve readers' performance. Note that despite senior authors YXJW and DD did not have face-toface interaction, they achieved a very good agreement which appears to be even better than the results of the inter-reader agreements reported by $\mathrm{Wu}$ et al. (34) from the University of California San Francisco (USA) group who conceived the original GSQ criteria. We believe this re-emphasizes the importance of well-defined measurement method. Our 
results also suggest that for the 6 young general radiologists to achieve the performance of the senior authors, a much longer period of coaching may be required. It has been noted that $\mathrm{eSQ}$ minimal and mild grade OVDs are often under-recognized or missed in clinical practice (36), while after self-leaning the number of missed minimal and mild grade OVDs by the six learner reader were very few in this study. Thus, in addition to research purpose, Wáng et al.'s article (18) discussed in this study may also service as a teaching material for clinical radiologists reading spine radiographs. Osteoporosis is a growing problem in the aging population, it is estimated that up to one in three women and one in five men over 50 years of age are affected (37), leading to millions of fractures annually and contributing substantially to morbidity and mortality. Subclinical OVF is an early sign of osseous fragility (4).

There are a few limitations for this study. A ground truth for OVD diagnosis and grading does not exist. Thus, the reference reading could still have suffered from subjectivity. To avoid potential controversies, readers were not asked to specifically annotate ECF sign, though we did ask readers to grade normal height VB with apparent ECF signs as minimal grade. We consider the issue of inter-reader agreement of ECF is beyond the scope of the current study, but it does have an implication in our choosing the phrasing of OVD rather OVF. In assessing a vertebra, there are usually three stepwise questions: (I) does a VD exist? (II) is this VD likely an OVD? (III) can this OVD be called OVF? We chose to use OVD in this paper as some of the minimal OVDs may not have clinical sequence $(32,33)$. Some authors suggested that OVD is OVF only when diagnosed on the basis of evidence or findings of endplate, cortical, or trabecular damage $(13,38)$. Imaging research is constrained by the investigators' vigilance. There is a possibility that if the participants of this study felt they were the PI (principle investigator) and fully responsible for the study (rather than being a participant), their performance might have been even better. Another point of note is that reader C2 had performed a study on traumatic VF and thus might have better knowledge of VF (39). However, during the 1st round of testing reading, reader C2 did not perform notably better than reader $\mathrm{C} 1$ and $\mathrm{C} 3$, thus unlikely this would have majorly affected the results.

In conclusion, this study tested the performance of evaluating OVD in 44 elderly women's spine radiograph by young general radiologists who did not have former experience in systematically evaluating OVD, and demonstrated good performance by these six learner readers after reading a few key references. Missed OVD were mostly minimal grade, missed mild grade OVD was very few; and false positive rate was also low. Our results are remarkably good considering that it is generally perceived that, without training with experienced readers, SQ criteria is difficult to apply. Our results confirm that it is important to publish standardized reference materials so that radiology readers can read and apply the same OVD criteria and derive consistent readings.

\section{Acknowledgments}

Funding: This study was partially supported by ITF project of Hong Kong SAR (code: ITS/334/18).

\section{Footnote}

Data Sharing Statement: Available at http://dx.doi. org/10.21037/jtd-20-2379

Conflicts of Interest: All authors have completed the ICMJE uniform disclosure form (available at http://dx.doi. org/10.21037/jtd-20-2379). The authors have no conflicts of interest to declare.

Ethical Statement: The authors are accountable for all aspects of the work in ensuring that questions related to the accuracy or integrity of any part of the work are appropriately investigated and resolved. This study was approved by the local ethics committees of authors' institutions. Informed consent for additional analysis of radiographs in this study was waived.

Open Access Statement: This is an Open Access article distributed in accordance with the Creative Commons Attribution-NonCommercial-NoDerivs 4.0 International License (CC BY-NC-ND 4.0), which permits the noncommercial replication and distribution of the article with the strict proviso that no changes or edits are made and the original work is properly cited (including links to both the formal publication through the relevant DOI and the license). See: https://creativecommons.org/licenses/by-nc-nd/4.0/.

\section{References}

1. Szulc P, Bouxsein ML. Overview of osteoporosis: epidemiology and clinical management. Vertebral Fracture Initiative Resource Document. Nyon, 
Switzerland: International Osteoporosis Foundation; 2011. PART I. p1-65.

2. Ismail AA, O'Neill TW, Cooper C, et al. Mortality associated with vertebral deformity in men and women: results from the European Prospective Osteoporosis Study (EPOS). Osteoporos Int 1998;8:291-7.

3. Broy SB. The vertebral fracture cascade: etiology and clinical implications. J Clin Densitom 2016;19:29-34.

4. Schwartz EN, Steinberg D. Detection of vertebral fractures. Curr Osteoporos Rep 2005;3:126-35.

5. Szulc P. Vertebral fracture: diagnostic difficulties of a major medical problem. J Bone Miner Res 2018;33:553-9.

6. Genant HK, Wu CY, van Kuijk C, et al. Vertebral fracture assessment using a semiquantitative technique. J Bone Miner Res 1993;8:1137-48.

7. Genant HK, Jergas M. Assessment of prevalent and incident vertebral fractures in osteoporosis research. Osteoporos Int 2003;14:S43-55.

8. Sugita M, Watanabe N, Mikami Y, et al. Classification of vertebral compression fractures in the osteoporotic spine. J Spinal Disord Tech 2005;18:376-81.

9. Jiang G, Eastell R, Barrington NA, et al. Comparison of methods for the visual identification of prevalent vertebral fracture in osteoporosis. Osteoporos Int 2004;15:887-96.

10. Wáng YXJ, Che-Nordin N. Some radiographically 'occult' osteoporotic vertebral fractures can be evidential if we look carefully. Quant Imaging Med Surg 2019;9:1992-5.

11. Lentle BC, Berger C, Probyn L, et al. Comparative analysis of the radiology of osteoporotic vertebral fractures in women and men: cross-sectional and longitudinal observations from the Canadian Multicentre Osteoporosis Study (CaMos). J Bone Miner Res 2018;33:569-79.

12. Wáng YX, Che-Nordin N, Deng M, et al. Osteoporotic vertebral deformity with endplate/cortex fracture is associated with higher further vertebral fracture risk: the Ms. OS (Hong Kong) study results. Osteoporos Int 2019;30:897-905.

13. Lentle B, Trollip J, Lian K. The Radiology of Osteoporotic Vertebral Fractures Redux. J Clin Densitom 2016;19:40-7.

14. Lentle B, Koromani F, Brown JP, et al. The Radiology of Osteoporotic Vertebral Fractures Revisited. J Bone Miner Res 2019;34:409-18.

15. Fechtenbaum J, Briot K, Paternotte S, et al. Bone section of the French Rheumatology Society. Difficulties in the diagnosis of vertebral fracture in men: agreement between doctors. Joint Bone Spine 2014;81:169-74.

16. Diacinti D, Vitali C, Gussoni G, et al. Misdiagnosis of vertebral fractures on local radiographic readings of the multicentre POINT (prevalence of osteoporosis in INTernal medicine) study. Bone 2017;101:230-5.

17. Messina C, Albano D, Rapisarda S, et al. Misdiagnosis of vertebral fractures on plain films: Are radiologists really working so bad? Bone 2017;105:307.

18. Wáng YX, Diacinti D, Yu W, et al. Semi-quantitative grading and extended semi-quantitative grading for osteoporotic vertebral deformity: a radiographic image database for education and calibration. Ann Transl Med 2020;8:398.

19. Yoshida T, Nanba H, Mimatsu K, et al. Treatment of osteoporotic spinal compression fractures. Conservative therapy and its limitation. Clin Calcium 2000;10:53-8.

20. Deng M, Kwok TCY, Leung JCS, et al. All osteoporotically deformed vertebrae with $>34 \%$ height loss have radiographically identifiable endplate/cortex fracture. J Orthop Translat 2018;14:63-6.

21. Wáng YX, Che-Nordin N, Deng M, et al. Elderly males with or without existing osteoporotic vertebral fracture have much lower future vertebral fracture risk than elderly females: the MrOS (Hong Kong) year-4 follow-up spine radiograph study. Osteoporos Int 2019;30:2505-14.

22. Wáng YX, Che-Nordin N. Informed communication with study subjects of radiographically detected osteoporotic vertebral deformity. Quant Imaging Med Surg 2018;8:876-80.

23. Wáng YX, Santiago RF, Deng $M$, et al. Identifying osteoporotic vertebral endplate and cortex fractures. Quant Imaging Med Surg 2017;7:555-91.

24. Wáng YX, Deng M, He LC, et al. Osteoporotic vertebral endplate and cortex fractures: a pictorial review. J Orthop Translat 2018;15:35-49.

25. Lentle BC. Gender and the recognition of vertebral fractures. Quant Imaging Med Surg 2020;10:1401-7.

26. Wáng YX, Wang XR, Che-Nordin N, et al. On the possibility of over-diagnosis of osteoporotic vertebral fracture at mid-thoracic level. J Thorac Dis 2019;11:5708-11.

27. Leidig-Bruckner G, Limberg B, Felsenberg D, et al. Sex difference in the validity of vertebral deformities as an index of prevalent vertebral osteoporotic fractures: a population survey of older men and women. Osteoporos Int 2000;11:102-19.

28. Kim YJ, Chae SU, Kim GD, et al. Radiographic Detection of Osteoporotic Vertebral Fracture without Collapse. J Bone Metab 2013;20:89-94.

29. Eastell R, Cedel SL, Wahner HW, et al. Classification of vertebral fractures. J Bone Miner Res 1991;6:207-15. 
30. McCloskey EV, Spector TD, Eyres KS, et al. The assessment of vertebral deformity: a method for use in population studies and clinical trials. Osteoporos Int 1993;3:138-47.

31. The International Osteoporosis Foundation (IOF): What We Do. Available online: https://www.iofbonehealth.org/ what-we-do/training-and-education/educational-slide-its/ vertebral-fracture-teaching-program, accessed on June 17, 2020.

32. Lentle B, Cheung AM, Hanley DA, et al. Scientific Advisory Council of Osteoporosis Canada. Osteoporosis Canada 2010 guidelines for the assessment of fracture risk. Can Assoc Radiol J 2011;62:243-50.

33. Johansson H, Oden A, McCloskey EV, et al. Mild morphometric vertebral fractures predict vertebral fractures but not non-vertebral fractures. Osteoporos Int 2014;25:235-41.

34. Wu CY, Li J, Jergas M, et al. Comparison of semiquantitative and quantitative techniques for the

Cite this article as: Wáng YXJ, Liu WH, Diacinti D, Yang DW, Iannacone A, Wang XR, Kripa E, Che-Nordin N, Diacinti D. Diagnosis and grading of radiographic osteoporotic vertebral deformity by general radiologists after a brief self-learning period. J Thorac Dis 2020;12(9):4702-4710. doi: 10.21037/jtd-202379 assessment of prevalent and incident vertebral fractures. Osteoporos Int 1995;5:354-70.

35. Buckens CF, de Jong PA, Mol C, et al. Intra and interobserver reliability and agreement of semiquantitative vertebral fracture assessment on chest computed tomography. PLoS One 2013;8:e71204.

36. Du MM, Che-Nordin N, Ye PP, et al. Underreporting characteristics of osteoporotic vertebral compressive fracture in back pain clinic patients of a tertiary hospital in China. J Orthop Translat 2019;23:152-8.

37. Keen RW. Burden of osteoporosis and fractures. Curr Osteoporos Rep 2003;1:66-70.

38. Wáng YX. A modified semi-quantitative (mSQ) grading scheme for osteoporotic vertebral fracture in elderly women. Quant Imaging Med Surg 2019;9:146-50.

39. Wang XR, Xu FR, Huang QL, et al. Radiological features of traumatic vertebral endplate fracture: an analysis of 194 cases with 263 vertebral fractures. Chin Med J (Engl) 2020. [Epub ahead of print]. 